\title{
MORTALIDADE DOS PARASITÓIDES Spilochalcis morleyi (HYMENOPTERA, CHALCIDIDAE) E Xanthozona melanopyga (DIPTERA, TACHINIDAE) EM PUPAS FEMININAS E MAS- CULINAS DE Brassolis sophorae (LEPIDOPTERA, NYMPHALIDAE)
}

\author{
RUSZCZYK A. ${ }^{1}$ e RIBEIRO J. C. ${ }^{2}$ \\ ${ }^{1}$ C.P. 9011, CEP 90050-110, Porto Alegre, RS \\ ${ }^{2}$ Departamento de Ecologia, Instituto de Biociências, UNESP, C.P. 199, CEP 13506-900, Rio Claro, SP \\ Correspondência para: Júlio César Ribeiro, Departamento de Ecologia, Instituto de Biociências, \\ UNESP, C.P. 199, CEP 13506-900, Rio Claro, SP, e-mail: juliocr@life.ibrc.unesp.br \\ Recebido em 29/07/98 - Aceito em 02/07/98 - Distribuído em 30/11/98
}

\section{ABSTRACT \\ Mortality of the parasitoids Spilochalcis morleyi and Xanthozona melanopyga in male and female pupa of Brassolis sophorae}

The mean number of adults emerged, number of death immatures, and the frequency of death of entire brood of the parasitoids Spilochalcis morleyi (Hymenoptera, Chalcididae) and Xanthozona melanopyga (Diptera, Tachinidae) in male and female pupa of the host, Brassolis sophorae (Lepidoptera, Nymphalidae) were compared. The number of emerged adults (Spilochalcis) was significantly higher in female pupa of the host. The number of death immatures per pupa was similar between the sexes of B. sophorae. However, a significantly higher frequency of death of the entire brood of both parasitoids was observed in female pupa of the host.

Key words: Brassolis sophorae, Spilochacis morleyi, Xanthozona melanopyga, host quality, parasitoid mortality.

\section{RESUMO}

Comparou-se o número médio de adultos emergidos, número de imaturos mortos e a freqüência com que ocorre a morte de toda a prole nos parasitóides Spilochalcis morleyi (Hymenoptera, Chalcididae) e Xanthozona melanopyga no hospedeiro, Brassolis sophorae (Lepidoptera, Nymphalidae). O número de parasitóides (Spilochalcis) que completaram seu desenvolvimento e emergiram foi significativamente maior em pupas femininas do hospedeiro. O número médio de imaturos de Spilochalcis mortos por pupa não diferiu entre os sexos do hospedeiro. Entretanto, nas pupas femininas de B. sophorae ocorreu uma freqüência significativamente maior de morte de toda a prole em ambas as espécies de parasitóides.

Palavras-chave: Brassolis sophorae, Spilochalcis morleyi, Xanthozona melanopyga, qualidade do hospedeiro, mortalidade de parasitóides.

\section{INTRODUÇÃO}

O lepidóptero Brassolis sophorae (L.,1758) é considerado uma praga de palmeiras cultivadas (Lordello, 1949; Rai, 1973). As larvas desta espécie são gregárias e têm atividade restrita ao crepúsculo, permanecendo inativas e reunidas em um abrigo de seda nos outros períodos do dia ou da noite. Brassolis sophorae apresenta um acentuado dimorfismo sexual de tamanho, tendo a fêmea adulta, em média, o dobro do peso do macho (Ruszczyk \& Carvalho, 1993). O dimorfismo de tamanho é também evidente nas pupas desta espécie. Spilochalcis morleyi (Ashmead, 1904) e 
Xanthozona melanopyga (Wiedemann, 1830) são endoparasitóides de B. sophorae (Gonzaga \& Lordello, 1952). Em X. melanopyga, apenas um indivíduo se desenvolve em cada pupa hospedeira, enquanto Spilochalcis é um parasitóide gregário. Ambas as espécies parasitam uma fração semelhante de pupas masculinas e femininas de B. sophorae (Ruszczyk, 1996). As pupas de B. sophorae parasitadas por estes dois inimigos naturais, têm em seu interior vestígios orgânicos do desenvolvimento dos parasitóides que permitem estimar o número e a mortalidade dos mesmos. A estimativa da mortalidade de parasitóides em condições de campo auxilia o entendimento da relação hospedeiro-parasitóide, entretanto, existem poucos trabalhos com essa abordagem: Moreira \& Becker (1987), por exemplo, determinaram a mortalidade de parasitóides de Nezara viridula em campo.

Nesta pesquisa comparamos a mortalidade dos imaturos de $S$. morleyi e $X$. melanopyga em pupas masculinas e femininas de B. sophorae.

\section{MATERIAL E MÉTODOS}

Pupas de Brassolis sophorae parasitadas por Xanthozona melanopyga ou Spilochalcis morleyi foram coletadas na área urbana de Campinas-SP $\left(22^{\circ} 55^{\prime} \mathrm{S}, 47^{\circ} 03^{\prime} \mathrm{W}\right)$, no período de setembro de 1985 a julho de 1987 . Os procedimentos de coleta estão descritos em Ruszczyk (1996). As pupas de B. sophorae foram classificadas segundo o sexo: a presença (fêmea) ou ausência (macho) de uma fenda transversal na porção ventral do penúltimo segmento abdominal é um caráter sexual. As pupas parasitadas por Xanthozona ou Spilochalcis foram seccionadas na ligação do tórax com o abdômen e seus restos orgânicos removidos com agulhas histológicas. Quando estes restos não continham vestígios de artrópodos detritívoros ou inquilinos, procedia-se à contagem do número de mêconios, larvas, pupas e pré-imagos mortos de $S$. morleyi que continham. A presença de um mêconio indica o desenvolvimento completo de um parasitóide.

Entre as pupas de B. sophorae parasitadas, observaram-se algumas em que todos os parasitóides morreram antes de completar seu desenvolvimento, faltando o orifício de emergência dos imagos dos parasitóides. Estes casos foram classificados de acordo com seus diferentes agentes causadores (Tabela 1). As frequiências destes diferentes tipos de mortalidade total em pupas do sexo masculino e feminino foram comparadas por testes de qui-quadrado.

O número médio de imaturos de $S$. morleyi, mortos em hospedeiros masculinos e femininos, e o número médio de indivíduos que completaram seu desenvolvimento em cada pupa foram comparados com testes $t$ de "Student". Os dados relativos ao número de pré-imagos e pupas mortos foram elevados ao quadrado para fins de normalização.

\section{RESULTADOS E DISCUSSÃO}

Spilochalcis morleyi foi observado ovipondo preferencialmente em pré-pupas e pupas de $B$. sophorae recém-formadas. No caso de Xanthozona melanopyga, provavelmente a fêmea oviposita diretamente nas larvas em adiantado estado de desenvolvimento (Mariconi \& Zamith, 1954). Observou-se (através da abertura do abrigo por onde saem as fezes das larvas), em uma palmeira de um jardim residencial na cidade de Campinas, uma fêmea deste parasitóide pousada na superfície interna do abrigo das larvas. Esta fêmea pousara cinco centímetros atrás das últimas larvas do grupo e, aparentemente, estava percebendo-as, já que seu corpo estava alinhado em direção às larvas. Relata-se esta observação, uma vez que não há na literatura referências sobre o comportamento de ataque ao hospedeiro por este parasitóide em condições naturais.

A mortalidade total da prole foi significativamente maior tanto para $S$. morleyi $\left(\chi^{2}=57.939\right.$, $\mathrm{gl}=1, P<0,001)$ como para $X$. melanopyga $\left(\chi^{2}=6.177, \mathrm{gl}=1, P=0,012\right)$ em pupas fêmeas de $B$. sophorae do que nas de sexo masculino (Tabela 1). As causas destas diferenças provavelmente estão relacionadas às características intrínsecas das pupas de cada sexo e sua identificação demanda estudos futuros.

Em S. morleyi, uma fração significativamente maior de morte de toda prole devido a sarcofagídeos ocorreu em pupas do sexo feminino, enquanto nas pupas de machos o parasitismo tardio, infecção por fungos e causas desconhecidas foram significantemente maiores (Tabela 1). Fuester \& Taylor (1996) sugerem que a maior mortalidade de parasitóides em pupas de machos de Lymantria dispar (Lep., Lymantriidae), causada por sarcofagídeos, está relacionada a maior 


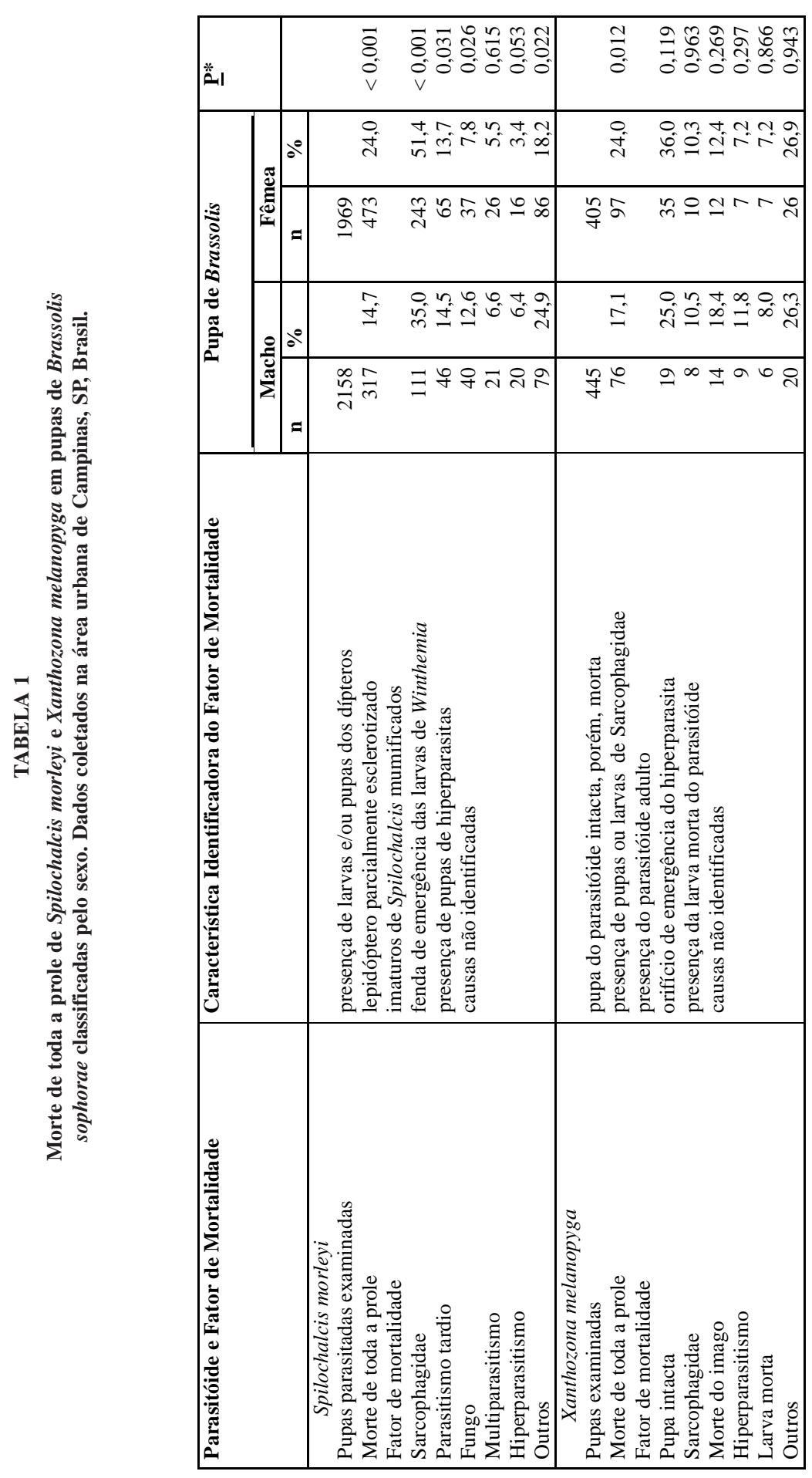


parasitismo nesse sexo, porque os dípteros usariam os orifícios feitos pelas vespas nas posturas para penetrar nas pupas. Para B. sophorae, o mesmo argumento não se aplica, já que não há diferença significativa no parasitismo de $S$. morleyi nos dois sexos (Ruszczyk, 1996).

As frequiências dos diferentes tipos de morte total da prole de $X$. melanopyga não diferiram significativamente entre os sexos do hospedeiro, embora nas pupas fêmeas do hospedeiro a freqüência da mortalidade total tenha sido maior. Nas duas espécies de parasitóides a morte de toda a prole devido a dípteros sarcofagídeos foi o tipo mais freqüente. Na literatura são freqüentes os relatos de mortalidade de himenópteros parasitóides devido a sarcofagídeos (Campbell, 1963; Stark \& Harper, 1982). Segundo Campbell (1963), as estimativas da efetividade de himenópteros como possíveis agentes de controle de lepidópteros podem estar subestimadas pela mortalidade causada pelos dípteros. Este autor observou que as larvas dos sarcofagídeos utilizavam o orifício de oviposição feito pelos himenópteros para penetrar na pupa do lepidóptero.
Em muitos casos de morte de toda a prole de S. morleyi, classificados como causa não identificada (Tabela 1), os parasitóides se encontravam na fase de pré-imago ou imago prestes a emergir, e morreram pouco antes do período em que emergiriam do hospedeiro. Nestes casos, a morte dos imaturos pode ter ocorrido devido a fatores abióticos geradores de estresse fisiológico, uma vez que não se verificou a presença de outros macrorganismos entomófagos que poderiam estar competindo por alimento ou modificando a qualidade do hospedeiro.

A sincronia no desenvolvimento demonstrou-se muito importante para a sobrevivência coletiva dos parasitóides dentro da pupa do hospedeiro. Isto porque, em muitos casos de mortalidade total da prole, a maioria dos indivíduos estava na fase pupal e alguns na fase larval, ou a grande maioria era pré-imago com alguns indivíduos na fase pupal.

A ocorrência de diferentes estágios de desenvolvimento de $S$. morleyi em um mesmo hospedeiro, além de refletir diferenças individuais, pode indicar também uma maior competição intra-

TABELA 2

Viabilidade e mortalidade dos imaturos de Spilochalcis morleyi em pupas de Brassolis sophorae classificadas pelo sexo. Dados coletados na área urbana de Campinas, SP, Brasil.

\begin{tabular}{|c|c|c|c|}
\hline Mortalidade dos Imaturos de Spilochalcis & \multicolumn{2}{|c|}{ Pupa de Brassolis } & $P^{*}$ \\
\hline & Macho & Fêmea & \\
\hline Pupas de $\boldsymbol{B}$. sophorae examinadas $\left(\boldsymbol{n}^{\underline{o}}\right)$ & 226 & 265 & \\
\hline № de mêconios (média \pm ep) & $45,13 \pm 1,21$ & $60,62 \pm 1,53$ & $<0,001$ \\
\hline № (mínimo - máximo) & $5-111$ & $9-155$ & \\
\hline \multicolumn{4}{|l|}{ Pré-imagos mortos } \\
\hline Pupas de $\boldsymbol{B}$. sophorae examinadas $\left(\boldsymbol{n}^{\underline{o}}\right)$ & 104 & 87 & \\
\hline № de pré-imagos mortos (média \pm ep) & $7,69 \pm 1,10$ & $8,59 \pm 1,49$ & 0,221 \\
\hline № (mínimo - máximo) & $1-65$ & $1-80$ & \\
\hline \multicolumn{4}{|l|}{ Pupas mortas } \\
\hline Pupas de $\boldsymbol{B}$. sophorae examinadas $\left(\boldsymbol{n}^{\underline{\boldsymbol{o}}}\right)$ & 140 & 126 & \\
\hline № de pupas mortas (média \pm ep) & $9,73 \pm 1,14$ & $8,14 \pm 0,98$ & 0,200 \\
\hline № (mínimo - máximo) & $1-100$ & $1-73$ & \\
\hline \multicolumn{4}{|l|}{ Larvas mortas } \\
\hline pupas de $\boldsymbol{B}$. sophorae examinadas $\left(\boldsymbol{n}^{\underline{\underline{o}}}\right)$ & 26 & 20 & \\
\hline № larvas mortas (média \pm ep) & $9,81 \pm 2,02$ & $6,85 \pm 2,16$ & 0,163 \\
\hline № (mínimo - máximo) & $1-45$ & $1-35$ & \\
\hline
\end{tabular}

* Significância das diferenças entre os sexos de B. Sophorae nas médias de parasitóides mortos, comparados por teste $t$. 
hospedeiro. A competição pode ser conseqüência da alocação de uma prole maior do que o hospedeiro pode sustentar pela ocorrência de autosuperparasitismo ou de superparasitismo por outra fêmea.

As proles de $S$. morleyi produzidas em pupas fêmeas foram maiores do que aquelas de pupas do sexo masculino (Tabela 2$)(t=-7.745, \mathrm{gl}=$ 489, $P<0,001)$. O tamanho da prole é definido pelo parasitóide no momento da oviposição, com base na qualidade e na disponibilidade dos hospedeiros (Vinson, 1976; Ikawa \& Okabe, 1985; Waage, 1986; Wellings, 1991). A diferença marcante de peso entre os sexos da pupa de $B$. sophorae pode ser interpretada como uma diferença de qualidade para o parasitóide.

Nos parasitóides pupais ou larvais-pupais, em que o alimento é fator limitante para o desenvolvimento da prole, uma vez que induções de mudança no comportamento alimentar do hospedeiro não ocorram, grandes proles podem causar maior competição entre os imaturos e aumentar a taxa de mortalidade entre eles (Godfray, 1994). Spilochalcis morleyi parece adequar o tamanho da prole à qualidade do hospedeiro e, como conseqüência a mortalidade média dos seus imaturos verificada nesta pesquisa não diferiu significativamente entre os sexos do hospedeiro (Tabela 2), apesar das diferenças de peso existentes entre eles.

Agradecimentos - Agradecemos a Gilson R. P. Moreira pela leitura crítica do manuscrito.

\section{REFERÊNCIAS BIBLIOGRÁFICAS}

CAMPBELL, R.W., 1963, Some ichneumonid-sarcophagid interactions in the gypsy moth Porthetria dispar (L.) (Lepidoptera: Lymantriidae). Can. Ent., 95: 337-345.

FUESTER, R. W. \& TAYLOR, P. B., 1996, Differential mortality in male and female gypsy moth (Lepidoptera: Lymantriidae) pupae by invertebrate natural enemies and other factors. Environ. Entomol., 25: 536-547.
GODFRAY, H. C. J., 1994, Parasitoids Behavioral and Evolutionary Ecology, Princenton, 473p.

GONZAGA, L. \& LORDELLO, L. G. E., 1952, Sobre os inimigos naturais da lagarta das palmáceas Brassolis sophorae sophorae (L.) (Lep., Brassolidae). An. Esc. Sup. Agric. "L. Queiroz", 9: 23-30.

IKAWA, T. \& OKABE, H., 1985, Regulation of egg number per host to maximize the reprodutive success in the gregarious parasitoid, Apanteles glomeratus L. (Hymenoptera: Braconidae). Appl. Ent. Zool., 20: 331-339.

LORDELLO, L. G. E., 1949, Brassolis sophorae lurida Stich, séria praga das palmáceas. Sit. e Faz. São Paulo, 14: 9-17.

MARICONI, F. A. M. \& ZAMITH, A. P. L., 1954, Contribuição para o conhecimento da Brassolis sophorae (Linnaeus, 1758), B. astyra (Godart, 1821) e de seus inimigos naturais. An. Esc. Sup. Agric. "L. Queiroz", sep. 212: $159-222$.

MOREIRA, G. R. P \& BECKER, M., 1987, Mortalidade, no período de pré-emergência, de parasitóides de Nezara viridula (Linnaeus, 1758) (Heteroptera: Pentatomidae), no estágio de ovo na cultura da soja. An. Soc. Ent. Brasil., 16: 297-313.

RAI, B. K., 1973, Brassolis sophorae and Castnia daedalus: chemical control of these major pests of coconut in Guyana. J. Econ. Ent., 66: 177-180.

RUSZCZYK, A. \& CARVALHO, M. C. Jr., 1993, Malfunction of ecdysis and female biased mortality in urban Brassolis sophorae (Nymphalidae: Brassolinae). J. Lepid. Soc. 47: 134-139.

RUSZCZYK, A., 1996, Spatial patterns in pupal mortality in urban palm caterpillars. Oecologia , 107: 356-363.

STARK, E. J. \& HARPER, J. D., 1982, Pupal mortality in forest tent caterpillar (Lepidoptera: Lasiocampidae): causes and impact on populations in southwestern Alabama. An. Ent. Soc. Am, 11: 1071-1077.

VINSON, S. B., 1976, Host selection by insect parasitoids. Ann. Rev. Entomol., 21: 397-419.

WAAGE, J. K., 1986, Family planning in parasitoids: adaptive patterns of progeny and sex allocation. In: Insect Parasitoids, J. K. Waage \& D. J. Greathead (eds.), Academic Press.

WELLINGS, P. W., 1991, Host location and oviposition on animals. In: Reproductive Behaviour of Insects, W. J. Bailey \& Ridsdill-Smith (eds.), Chapmam \& Hall. 